Iustitia Socialis. Revista Arbitrada de Ciencias Jurídicas

Año VI. Vol. VI. N 1. Edición Especial. 2021

Hecho el depósito de Ley: FA2016000064 ISSN: 2542-3371

FUNDACIÓN KOINONIA (F.K). Santa Ana de Coro, Venezuela

Marcos Antonio Portilla-Kirby; Andrés Esteban Jaimes-Grimaldos

http://dx.doi.org/10.35381/racji.v6i1.1463

\title{
El consentimiento legítimo en la práctica anestésica
}

\section{Legitimate consent in anesthetic practice}

\author{
Marcos Antonio Portilla-Kirby \\ marcos.portilla.78@est.ucacue.edu.ec \\ Universidad Católica de Cuenca, Cuenca \\ Ecuador \\ https://orcid.org/0000-0002-5699-1187 \\ Andrés Esteban Jaimes-Grimaldos \\ andres.jaimes@ucacue.edu.ec \\ Universidad Católica de Cuenca, Cuenca \\ Ecuador \\ https://orcid.org/0000-0002-4779-1239
}

Recepción: 15 de septiembre 2021

Revisado: 25 octubre 2021

Aprobación: 15 de noviembre 2021

Publicación: 01 de diciembre 2021 


\title{
RESUMEN
}

En el presente artículo se parte de la conceptualización del procedimiento anestésico como un acto médico complejo expuesto a los riesgos de toda intervención farmacológica e instrumental, capaz de producir secuelas que van desde efectos leves hasta consecuencias fatales, por lo cual se propone una estrategia para mejorar su aplicación y legitimar su contenido con un formato de consentimiento que reúna las condiciones de legitimidad y legalidad que pueda servir como referente a que los médicos anestesiólogos públicos y privados del Sistema de Salud.

Descriptores: Ética de la ciencia; bioética; ética médica; deontología. (Tomado del tesauro de la UNESCO).

\begin{abstract}
This article starts from the conceptualization of the anesthetic procedure as a complex medical act exposed to the risks of all pharmacological and instrumental interventions, capable of producing sequelae that range from mild effects to fatal consequences, for which a strategy is proposed to improve its application and legitimize its content with a consent format that meets the conditions of legitimacy and legality that can serve as a reference for public and private anesthesiologists of the Health System.
\end{abstract}

Descriptors: Éthics of science; bioethics; medical ethics; deontology. (Taken from the UNESCO thesaurus). 


\section{INTRODUCCIÓN}

El consentimiento informado, nombre con que en el ejercicio médico se denomina al documento por medio del cual el paciente manifiesta su voluntad para someterse a un tratamiento generalmente invasivo, es un instrumento originado en el Código de Núremberg en 1947, como uno de los requisitos indispensables que tiene que firmar todo paciente que va a intervenirse, sin embargo, hasta la actualidad no ha conseguido aplicarse como establece la normativa.

En experiencias intercambiadas en congresos de las especialidades médicas se percibe que hay dificultades para su plena ejecución, entre los principales: la falta de exigencia institucional de la obligatoriedad de su uso, diferencias en la práctica médica privada e institucional, una débil relación médico-paciente es débil y cierta falta de interés para eliminar las diferencias culturales que normalmente existen entre paciente y anestesiólogo.

Para los profesionales sanitarios en general y para las especialidades como la anestesiología -en particular-, este incumplimiento puede entenderse a través del hecho de que ciertas herramientas como el consentimiento fueron introducidas dentro de la práctica clínica en los primeros veinte años del presente siglo, período que al parecer resulta corto si se considera el hecho que no se dispone de un formato estandarizado para que al aplicarlo llene las inquietudes tanto del paciente como del anestesiólogo.

Esta carencia, constituye una afectación a la prestación de servicios médicos que han sido definidos en la actualidad como un proceso dinámico centrado en mejorar la atención elevando la calidad en forma continua lo que significa un cambio en los patrones culturales de la organización involucrando a todos sus actores para satisfacer las necesidades y preferencias de los pacientes (Forrellat-Barrios, 2014).

Esta propensión a disminuir los errores clínicos y administrativos significa estandarización de procedimientos uno de los cuales es, precisamente, la implementación del consentimiento. De experiencias conocidas, aunque no publicadas, se conoce que muchas instituciones sanitarias públicas y privadas o no practican el consentimiento de 
manera adecuada o no cuentan con un formato estandarizado que garantice realmente los derechos del paciente, resultando en la práctica una suerte de improvisación o más bien un arbitrio sujeto a la conveniencia institucional y a veces personal del profesional de la salud.

Frente a esta problemática, la presente investigación tuvo como finalidad caracterizar los actos anestésicos y sus riesgos, así como la naturaleza del consentimiento como derecho fundamental de los pacientes en términos de legitimidad y legalidad.

El primer segmento del desarrollo se ocupó de la parte teórica, describiendo las particularidades del acto anestésico, considerado como sustrato indispensable para comprender los elementos que definen el riesgo para el paciente sometido al intervencionismo médico; En un segundo momento, se sintetizaron las experiencias sobre la aplicación del consentimiento y se adentró en el análisis de su contenido, lo que permitió aportar una propuesta mediante el anexo al mismo, sobre cuál sería un documento modelo de consentimiento como instrumento que podría ser adoptado en la práctica clínica de la especialidad de anestesiología.

De esta manera, se pretende responder las interrogantes que generó la presente investigación: ¿Qué dificulta al anestesiólogo aplicar de forma eficiente el consentimiento? y ¿cuál debe ser su contenido para cumplir con los principios de la bioética, condición indispensable para el ejercicio médico actual?

\section{REFERENCIAL TEÓRICO}

\section{Procedimientos anestésicos}

Para fines de la presente investigación, se indicará que la anestesia reconoce dos clases de procedimientos y diversas modalidades en cada uno de ellos, a saber: 1) anestesia general y 2) anestesia locorregional. Ambos procedimientos anestésicos pueden producir riesgos con secuelas que van desde efectos leves hasta consecuencias fatales. 


\section{Del consentimiento informado al consentimiento legítimo}

\subsection{Antecedentes del consentimiento}

El consentimiento está estrechamente relacionado con un tipo específico de información, es decir para que exista consentimiento debe haber pleno conocimiento de lo que se está consintiendo (Chrimes \& Marshall, 2018). Todo paciente tiene derecho a ser informado 0 ilustrado de la manera más clara posible de su estado de salud y de las alternativas de tratamiento, puntualizando en ellas los beneficios y aun los riesgos que casi siempre son previsibles. Para ello debe utilizarse información concreta y veraz en un lenguaje comprensible que no dé lugar a preocupaciones innecesarias de tal manera que propicie la participación del enfermo en el tratamiento de su propia enfermedad y acepte otorgar el consentimiento (Waisel et al. 2009).

El principio del consentimiento, orientado a la licitud de la asistencia sanitaria, tiende a reflejar el concepto de autonomía y de autodeterminación decisional de la persona quien requiere y solicita intervenciones médicas y/o quirúrgicas. Esta fórmula jurídica, en los últimos años, ha ganado no sólo un espacio considerable, sino también una importancia fundamental en la elaboración y planteamientos doctrinales, así como en las interpretaciones jurídicas, influyendo así en la actividad cotidiana de la profesión médica (Stain, 2016).

El consentimiento sigue siendo objeto de continuas exploraciones, tanto en lo que se refiere al perfil teórico ya confirmado, como al ambiguo aspecto práctico y consecuente. Analizando cómo nació y se desarrolló el concepto y rol del consenso con las excursiones más adecuadas y razonables para hacerlo válido y obtenerlo, es imposible no tomar en consideración, por un lado, los orígenes filosóficos muy antiguos y, por otro, el hecho de que estuvo condicionada por la religión con los aspectos morales y la evolución deontológica acelerada con caminos paralelos a las necesidades y las necesidades de los avances que ofrecen las nuevas formas de tratamiento y las nuevas aplicaciones biotecnológicas (Mallardi, 2005). 
El principio del consentimiento es una condición relativamente nueva. De hecho, ya en tiempos de las civilizaciones egipcias, griegas y romanas, se han encontrado documentos que muestran cómo la intervención del médico tuvo, de alguna manera, la primera aprobación del paciente. Platón (ley IV) ya había previsto los problemas, los procedimientos y los modos de información que son, en síntesis, en la raíz de los principios de la presente fórmula de consentimiento y correlacionó la práctica de la información y el consenso con la calidad y posición social del paciente (Rose, 1986).

El médico hipocrático respetaba un principio de responsabilidad profesional más religioso y de tipo moral, pero, desde el punto de vista jurídico, muy débil en cuanto dependía de normas elaboradas por seres humanos. La convicción y certeza de que el médico actuó, en interés del bienestar de su paciente, se ha ido transmitiendo a lo largo de los siglos dotando al médico de autoridad moral y una especie de impunidad jurídica, condiciones que correspondían, casi como un reflejo, con el deber de obediencia y sujeción, por parte del paciente (Mallardi, 2005).

Como es bien sabido, el 19 de diciembre de 1946 se inició en Nuremberg un juicio de médicos nazis y se definió un código en el que los jueces, todos estadounidenses, enfatizaban claramente una visión de la investigación y la tecnología médica: la ciencia nunca debe transformar o considerar al ser humano como un instrumento para ser empleado con fines científicos (Mainetti, 1989). Una puntualización necesaria, antes de entrar en materia, constituye aclarar el porqué de la nomen iuris que se utilizará en el presente informe para referirnos al documento en cuestión.

El primer documento escrito en el que se hace alusión a su necesidad, surge en la

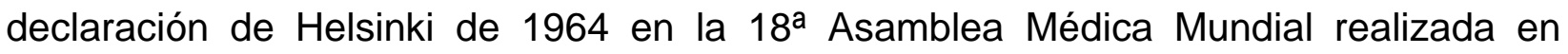
Finlandia y es revisada por la 29aㅡ Asamblea Médica Mundial de Tokio, Japón, en 1975. Posteriormente, ha sido ratificado como un instrumento indispensable para garantizar el cumplimiento de los principios éticos para las investigaciones médicas en seres

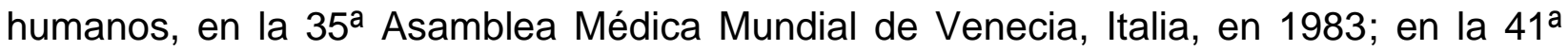
Asamblea Médica Mundial de Hong Kong, en 1989; en la 48 Asamblea General 
Somerset West de Sudáfrica, en 1996, y en la 52ª Asamblea General de Edimburgo, Escocia, en octubre de 2000 (Ramírez et al. 2006).

Las primeras publicaciones sobre su vigencia lo denominan consentimiento voluntario, pero luego se unifican criterios para su denominación y aparece en la literatura como consentimiento informado (Cocanour, 2017). Algunas comunidades médicas han propuesto denominaciones como consentimiento educado para casos en los que no se utilice un documento escrito y firmado, como ocurre en los procedimientos cumplidos en la consulta médica (Núñez de Villavicencio, 2006) y otros autores, conjugando los aspectos bioéticos y legales señalan que consentimiento idóneo o ilustrado definiría con más propiedad la función que cumple este documento (Córdoba, 2010).

Una modalidad del consentimiento informado constituye el asentimiento informado por el que los menores de edad asienten el procedimiento en presencia de sus padres o tutores y como una constancia complementaria al consentimiento que lo firman los responsables legales del paciente (Pinto \& Díaz, 2013).

\subsection{Obstáculos para la eficacia del consentimiento legítimo}

Entre las tareas del anestesiólogo, consta la obligación de informar y esto se logra con una buena relación anestesiólogo-paciente. No obstante, la relación que se entabla entre los anestesiólogos y el paciente, está matizada permanentemente de circunstancias unas muy cruciales y otras muy puntuales (Wilkinski \& Piaggio, 1993). En el marco indisoluble de la interacción cirugía-anestesia desde los años setentas del siglo pasado volvió a tener vigencia la denominada cirugía ambulatoria cuyo fundamento es permitir al paciente el retorno al hogar pocas horas después de realizado un procedimiento quirúrgico realizado con anestesia local o regional y hasta general, pero con cuidados postoperatorios sin internación (Cruz, 2020).

En este escenario la relación anestesiólogo paciente también ha sido menoscabada y no existe alternativa que ocupe su lugar aún dentro de la vigencia de los derechos del paciente (Nasiłowski, 2007). Visto así, la consulta preanestésica es un imperativo, no es 
un trámite sino una necesidad obligatoria (Castañeda, 2019), que debe ser implementada en el sistema nacional de salud de nuestro país, como la mejor forma de llenar un vacío en el ejercicio de la especialidad y elevar la calidad de atención al paciente quirúrgico.

\subsection{La bioética}

La ética clínica es la aplicación de teorías, principios, reglas y pautas éticas a situaciones clínicas en la medicina. Por tanto, ética clínica es análoga de medicina clínica en el sentido de que los principios y conceptos generales deben aplicarse de manera inteligente y reflexiva a circunstancias clínicas únicas (Narro-Nobles at al, 2004). Las tres principales teorías éticas son el consecuencialismo, por el cual las consecuencias de una acción determinan si es ética; la deontología, según la cual ser ético es cumplir con el deber, y la ética de la virtud, según la cual la ética es cuestión de cultivar las virtudes adecuadas (Torres, 2011).

En términos éticos, el médico debe defender cuatro principios fundamentales, como son la autonomía del paciente, la beneficencia, la no maleficencia y la justicia, por lo cual, cuando estos principios entran en conflicto, es fundamental acudir a los enfoques alternativos como la primacía de la beneficencia, la ética basada en el cuidado, la ética feminista y la ética narrativa, los cuales ayudan a definir las limitaciones del principialismo y brindan una perspectiva más amplia de la ética médica (Taylor, 2013).

\section{MÉTODO}

\section{Tipo De Investigación}

La presente propuesta investigativa se enmarcó dentro del tipo de investigación, jurídica - documental y descriptiva. Así pues, en lo concerniente con esta modalidad de investigación, García Dora, miembro del Sistema Nacional de Investigadores e investigadora del Instituto de Investigaciones Jurídicas de la Universidad Anáhuac México Norte; en su libro La metodología de la investigación jurídica en el siglo XXI, define la investigación jurídica como: 
Conjunto de actividades, llevadas a cabo por el jurista, que tienden a la búsqueda del derecho, de sus antecedentes $y$, por supuesto, de su actualidad y realidad. De esta forma, el derecho constituye el objeto de la investigación científica, y se convierte en investigación jurídica. Los fines de la investigación jurídica son ampliar, corregir, verificar o aplicar los conocimientos adquiridos, y es de suma importancia, ya que puede dar soluciones válidas a los problemas que afectan a la sociedad, o por lo menos, puede dar herramientas para comprenderlos mejor (García, 2015, p.454).

Por su parte, la investigación jurídica - documental es definida por Duarte \& Parra, en su libro: Lo que debes saber sobre un trabajo de investigación, como aquella que se apoya en "técnicas documentales se confronta la opinión del autor(a) con la de otros autores, como los avances que ha tenido el tema y conocer más del objeto de investigación, también recopila la mayor cantidad de información en el menor tiempo posible" (Duarte \& Parra, 2014).

Con relación a la investigación descriptiva, es definida como aquella que consiste en la caracterización de un hecho, fenómeno, individuo o grupo, con el fin de establecer su estructura o comportamiento. Se empleó esta investigación, porque a partir del estudio del análisis documental propuesto para la comprensión de los riesgos propios de los actos anestésicos y el derecho al consentimiento, se permitió identificar la importancia de una mejor práctica del mismo, en términos no sólo de legalidad, sino además de legitimidad.

\section{Técnicas y Procedimientos para la recolección de la Información}

Instrumentos. Para la presente investigación, se utilizaron referencias bibliográficas con los ítems de búsqueda referidos a los actos anestésicos y al consentimiento informado según los autores de este trabajo, legítimo-, los cuales permitieron conocer los factores determinantes del problema planteado. 
Fuente Primaria. Normatividad ecuatoriana e internacional y jurisprudencia de la Corte Interamericana de Derechos Humanos.

Fuente Secundaria. Para la presente investigación, se emplearon libros, archivos, artículos, informes y publicaciones que fueron realizados otros investigadores y doctrinantes sobre este tema.

\section{RESULTADOS}

Se describen los resultados desarrollados:

\section{Actos anestésicos y sus riesgos}

Con el advenimiento de nuevos compuestos medicamentosos utilizados en los procedimientos anestésicos y los nuevos dispositivos digitales producidos por la tecnología moderna -donde la informática juega un papel decisivo-, se ha alimentado la idea de que los riesgos asociados a la anestesia se han reducido.

No obstante, (Lagasse, 2002), publicó un estudio en el que encontró una tasa de mortalidad relacionada con la anestesia -según lo determinado por la revisión por pares, y el cual se mantuvo estable en la década previa al estudio, con aproximadamente 1 muerte por cada 13,000 procedimientos, motivo por el cual se viene investigando constantemente en busca de resultados concluyentes.

Dado que la anestesia no es un acto médico aislado, sino que se realiza como una condictio sine qua non para cualquier procedimiento intervencionista, los estudios para evaluar sus riesgos involucran otros elementos que a veces pueden ser más determinantes; es decir, la lesión, el daño o la mortalidad ocurridas alrededor de una intervención, siempre serán efectos de causas multifactoriales y no propiamente de la anestesia (Fleisher, 2016).

Los errores humanos, como la aplicación de una técnica inapropiada, falta de equipos especializados para una situación contingente, ausencia de información sobre las 
condiciones clínicas del paciente, inexperiencia del operador ante determinada maniobra, accidentes en la técnica anestésica, fallo de los equipos, errores en la comunicación, limitaciones de los estándares terapéuticos, hemorragias masivas impredecibles, falta de supervisión, agotamiento por exceso laboral del anestesiólogo han sido, entre otros, algunos de los factores identificados como riesgos que con frecuencia desembocan en accidentes anestésicos con desenlaces fatales (Lagasse, 2002).

Una división necesaria, aunque arbitraria, es considerar que la anestesia es de dos tipos: 1) anestesia general y 2) anestesia locorregional; en la cual, la primera involucra una afectación del sistema nervioso central con pérdida de la conciencia, hipnosis profunda y abolición de los reflejos; en tanto que la segunda, supone eliminación de la sensibilidad en un área delimitada -anestesia local-, en un miembro o región del cuerpo humano anestesia regional.

Sin embargo, de esta clasificación, en la práctica clínica toda anestesia locorregional o sedación complementaria cuando el paciente está despierto, puede transformarse en anestesia general, dependiendo de las necesidades del equipo quirúrgico; es decir, en una agresión sistémica impredecible que afecta al organismo en su conjunto (Mikstacki et al. 2015). Esta conversión, comúnmente está decidida por las necesidades del equipo quirúrgico y no menos frecuente por la necesidad del paciente que al requerir de una sedación complementaria, sufre fácilmente en una depresión del sistema nervioso central que afecta principalmente los reflejos del paciente.

El que la anestesia general no está exenta de toxicidad es una preocupación que ha generado múltiples investigaciones y se ha reportado que la exposición a los fármacos como anestésicos volátiles, midazolam, propofol, ketamina y óxido nitroso aumenta la muerte de las células neuronales en animales jóvenes como ratas, ratones y primates no humanos, y por esto, la posibilidad de que se produzca neurotoxicidad inducida por anestésicos en niños ha suscitado preocupaciones sobre la seguridad de la anestesia, pero hacen falta estudios para verificarlo (McCann \& Soriano, 2019). 
En cuanto a la toxicidad anestésica, es necesario aclarar que hay claras diferencias entre el tipo y la dosis de fármacos utilizados en procedimientos cortos o para cirugías no complejas, como ocurre con la anestesia local o regional, en las que casi siempre el paciente está despierto. En el ámbito médico-legal, y para fines de aplicación de un consentimiento legítimo, son las complicaciones del acto médico las que concitan el interés; así como las complicaciones, efectos colaterales o iatrogenias, que ocurren a cualquier paciente y en cualquier ambiente sanitario en el mundo, cuando resultan predecibles (Sivkov et al. 2019).

Los estándares de seguridad diseñados para eliminar los eventos anestésicos durante su administración, han sido ampliamente difundidos, socializados, aceptados y han merecido la aceptación de la comunidad de especialistas y su sistematización se debe a la Sociedad Americana de Anestesiólogos en adelante ASA, la cual se usa para determinar el estado físico del paciente quirúrgico por su condición biológica que, asociada a otros factores clínicos, es una herramienta útil para predecir riesgos (ASA, 2016), sin mencionar que la mayoría de países cuenta con normas de seguridad para el ejercicio de la especialidad dentro del sistema nacional de salud.

Históricamente, la Confederación Latinoamericana de Sociedades de Anestesiología en adelante CLASA, es un organismo gremial y científico de la especialidad en el subcontinente, encargada de difundir en 1995, a través de la Sociedad Colombiana de Anestesiología y Reanimación -en adelante SCARE- un documento elaborado por varios especialistas, para adecuarlo a las necesidades del país y sus similares confederados.

Este documento, denominado Normas Mínimas de Seguridad en Anestesiología, concuerda con los estándares de la Sociedad Americana de Anestesiólogos y similares europeas, que desde los años 80 del siglo pasado, vienen sistematizando el manejo de la especialidad en busca de mejores prácticas basadas en la investigación clínica (Haller et al. 2011). A partir de dicha época, los países han adoptado los estándares internacionales que se han establecido a nivel internacional, como el caso de Ecuador; $y$ 
en otros casos, han acondicionado sus propios estándares, como es el caso de Chile, Argentina, Brasil, Cuba, México y Venezuela (Hernández-Cortez, 2017).

Hacia el año 2010, se llevó a cabo el lanzamiento de la Declaración de Helsinki sobre la seguridad del paciente en anestesiología, lo que constituyó un gran paso para las iniciativas de seguridad del paciente en anestesiología europea y mundial. En virtud de ello, se han tomado varias medidas en mejoras continuas y la tarea para garantizar que cada paciente reciba la atención de anestesiología más segura posible, está en marcha (Mellin-Olsen \& Staender, 2014).

Sintetizando lo anterior, los riesgos de la anestesia general pueden ser: ataque cardíaco; insuficiencia cardíaca o ataque cerebral; aumento o disminución de la presión arterial; neumonía u otros problemas respiratorios; reacciones alérgicas a los medicamentos utilizados con choque anafiláctico; daño muscular e hipertermia maligna, parada cardíaca con isquemia cerebral, lesión cortical y muerte (Kounis et al. 2020), (Mesa et al. 2016), (Schönenberger et al. 2019).

Ahora, los riesgos que se asocian a la anestesia locorregional pueden ser: toxicidad neurológica en ramas o plexos nerviosos, con parálisis temporal o permanente tipo síndrome de cauda equina; hemorragia epidural con secuelas de parálisis permanente; lesión medular irreversible; arritmias; hipotensión; bradicardia; convulsiones; depresión del sistema nervioso central y muerte (Zheng et al. 2020), (Spector et al. 2008), (Pardina et al. 2001), (Lin et al. 2017).

No obstante, más importante que asignar un riesgo anestésico según el tipo de procedimiento quirúrgico, terapéutico o intervencionista, resulta fundamental asumir que el acto anestésico, constituye un riesgo en cualquiera de sus modalidades, pues tal como lo ha definido la ASA, la práctica anestésica involucra tanto (i) La evaluación y el tratamiento preoperatorio, intraoperatorio y posoperatorio de pacientes cuando resultan inconscientes y/o insensibles al dolor y el estrés emocional durante procedimientos quirúrgicos, obstétricos, radiológicos, terapéuticos y de diagnóstico u otros procedimientos médicos y participación en la coordinación general de la atención. (ii) La 
protección y mantenimiento de funciones vitales y órganos vitales, como el cerebro, corazón, pulmones, riñones, hígado, endocrino, integridad de la piel, nervios sensitivos y motores, debido al estrés por procedimientos anestésicos, quirúrgicos y otros procedimientos médicos, entre otros (ASA, 2021).

Adicionalmente, al período anestésico, debe llamarse la atención sobre la existencia de períodos preanestésicos y postanestésicos, los cuales amplían las responsabilidades del anestesiólogo, pues tal como lo sostuvieron, (Mena \& Rodríguez-Mota, 2014), en tales períodos deben cumplirse la evaluación y preparación preanestésica, la premedicación, la preparación y revisión de equipos, medicamentos, fluidos y suministros de gas; la disponibilidad del personal y el equipo de enfermería necesarios para una atención postanestésica segura; transferencia de información de atención pertinente a las necesidades específicas del paciente y una transición segura; permanencia con el paciente durante el tiempo médicamente necesario y hasta que el proveedor de atención médica que lo recibe tenga toda la información necesaria para asumir la atención, entre otros.

Inobservar algunos de los supuestos anteriores, hace que se incremente la condición de riesgo en el acto anestésico, aunque se hayan aplicado técnicamente las sustancias medicamentosas seleccionadas y se haya cumplido con el rigor de las destrezas requeridas, pues los eventos negativos anestésicos pocas veces ocurren por administración inadecuada de fármacos, estando más relacionados con los cuidados como factores organizacionales (Cordero, 2011).

Así las cosas, se debe comprender que el acto anestésico es un asunto complejo que involucra la intervención de profesionales en medicina, funcionarios sanitarios, condiciones ambientales, los cuales no siempre actúan en armonía y las probabilidades de los desenlaces serán siempre impredecibles, pues tal como lo señalaba Osler, los errores de juicio son inevitables en un arte como la anestesia, pues la misma consiste de manera esencial en balancear o evaluar probabilidades (Osler, 1913). 
Es en este contexto, surge el interés por manejar adecuadamente el consentimiento como la mejor herramienta para el anestesiólogo y para el paciente quien recibirá anestesia, aceptando el hecho de que, en medicina terapéutica, toda intervención farmacológica, y además instrumental, es capaz de producir secuelas en el organismo que van desde efectos leves hasta consecuencias fatales (Luethi \& Liechti, 2020).

\section{El consentimiento legítimo.}

Para el caso ecuatoriano, el Código de Ética Médica, actualizado el 17 de agosto de 1992, el artículo 15 hizo referencia al consentimiento en el sentido de que es necesario el pronunciamiento previo del enfermo para cualquier tipo de intervención quirúrgica (FME, 2021) y en 1995 cuando se crea la ley de Derechos y Amparo de los Pacientes, actualizada el 22 de diciembre de 2006, se ratificó este derecho.

La Asamblea Nacional de 2008 ratificó en el artículo 362 de la constitución: "Ios servicios de la salud serán seguros, de calidad y calidez y garantizarán el Consentimiento llustrado, el acceso a la información y la confidencialidad de la información de los pacientes (Asamblea Nacional de Montecristi, 2008).

A nivel del sistema interamericano la Corte Interamericana de Derechos Humanos establece que el consentimiento legítimo "es una obligación a cargo de las instituciones de salud, las personas mayores ostentan la titularidad de este derecho, sin embargo, se puede transferir bajo ciertas circunstancias a sus familiares o representantes. Asimismo, persiste el deber de informar a los pacientes o, en su caso cuando proceda, a sus representantes sobre los procedimientos y condición del paciente" (CIDH, 2020).

Establecido lo anterior, se debe afirmar que el anestesiólogo debe incluir en su ilustración dos aspectos: 1) la información que estará siempre en relación con lo que el paciente quiere saber y cuándo lo quiere saber, para contar con los elementos que le permitan adoptar una decisión consciente; en este punto, el no proporcionar la información correctamente se convierte en una forma de manipulación y su desconocimiento puede ser tan peligroso como la mala información, y 2) Que el paciente comprenda la 
información que se le está ilustrando, para lo cual el médico debe utilizar un lenguaje claro, preciso y adaptado al nivel sociocultural del enfermo, se debe omitir en lo posible terminología médica y en caso de hacerlo habrá que explicar su significado (Moctezuma, 2002), (Dobler, 2001).

Además, el consentimiento es un requisito indispensable para todo procedimiento intervencionista, por lo cual debe utilizarse para la aceptación de un diagnóstico, de un tratamiento o de participación en un protocolo de investigación. No cumplir este requisito expone al médico a quedar expuesto a una demanda judicial que podrá ser evitada si el médico prueba con base en el expediente clínico bien elaborado que no incurrió en negligencia, impericia o inobservancia de la normativa que rige el ejercicio de la medicina en general y de las especialidades quirúrgicas en particular (Miranda-Novales \& VillasísKeever, 2019).

Aun así, la firma del consentimiento, por sí solo, no excluye la posibilidad de un reclamo legal pero la documentación que comprende el consentimiento legítimo, constituye la mejor defensa en caso de las demandas de pacientes que fueron informados apropiadamente acerca del tratamiento programado y de las complicaciones o riesgos que pudieran presentarse, pues en caso contrario, el anestesiólogo será acusado de negligencia cuando estas recomendaciones se incumplan (Cocanour, 2017), (Gonzáles, 2004), (Zarnegar, 2018).

Desde el punto de vista legal, la relación que se establece entre el médico y el paciente, es entonces, una relación contractual y para que exista un contrato es imprescindible que exista esa aceptación que implica obligarse y adquirir derechos cuando se recibe una información adecuada acerca de lo que se pretende y admite, por parte de los sujetos de la relación jurídica, en este caso, el médico y el paciente, siendo el consentimiento esencial en todo contrato de salud (Wheeler, 2017), (Zúñiga \& Zúñiga-Hernández, 2019). Si el paciente consiente una acción sin haber tenido la información debidamente ilustrada, puede considerarse un engaño y si se produjere daños se podrá demandar su reparación, según lo que establece el código civil de cada país (García, 2001). Por esta razón, la idea 
de un consentimiento legítimo está orientada a la licitud de la asistencia sanitaria, la cual tiene a proteger el concepto de autonomía y de autodeterminación decisional de la persona que requiere y solicita intervenciones médicas y/o quirúrgicas, siendo esta fórmula jurídica, en los últimos años, la que ha ganado un espacio considerable y una importancia en la elaboración y planteamientos doctrinales, así como en las interpretaciones jurídicas, influyendo así en la actividad cotidiana de la profesión médica (Stain, 2016). El consentimiento legítimo sigue siendo objeto de continuas exploraciones, no sólo en lo que se refiere al perfil teórico ya confirmado; sino, en cambio, al ambiguo aspecto práctico y consecuente.

Analizando cómo nació y se desarrolló el concepto y rol del consenso con las excursiones más adecuadas y razonables para hacerlo válido y obtenerlo, es imposible no tomar en consideración, por un lado, los orígenes filosóficos muy antiguos y, por otro, el hecho de que estuvo condicionada por la religión con los aspectos morales y la evolución deontológica acelerada con caminos paralelos a las necesidades, los avances que ofrecen las nuevas formas de tratamiento y las nuevas aplicaciones biotecnológicas (Mallardi, 2005).

De otra parte, resulta fundamental generar la conciencia del deber a cargo del anestesiólogo de informar, lo cual se logra con una buena relación anestesiólogopaciente. No obstante, siguiendo a (Wilkinski \& Piaggio, 1993), está relación se ve afectada por diferentes aspectos circunstanciales como que:

(i) El paciente llega al procedimiento anestésico después de un recorrido que va desde el diagnóstico definitivo hasta la proposición quirúrgica y hasta ese momento nunca se ha relacionado con el anestesiólogo.

(ii) Muchos enfermos y familiares tienen la idea de que el anestesiólogo no es un médico o lo consideran como un médico general poseedor de ciertas destrezas y capacidades técnicas especiales.

(iii) La relación anestesiólogo-paciente no posee ni la continuidad ni el tiempo que sí se invierten en las demás acciones médicas, como hasta llegar al diagnóstico definitivo y 
planear una solución terapéutica -en la mayoría de casos el anestesiólogo conoce personalmente al paciente el día anterior a la cirugía, período muy corto para que la entrevista y la valoración sean adecuadas-.

(iv) La consulta preanestésica no se cumple en la medida que se recomienda y se convierte únicamente en ello, la que realiza generalmente el médico residente en formación de la especialidad y la experiencia señala que en estas situaciones el procedimiento se cumple con incertidumbre, con limitación de tiempo y en ambientes muchas veces inapropiados como la sala de operaciones (Wilkinski \& Piaggio, 1993).

Otro factor que incide de manera directa en una relación débil, es el hecho que, desde los años setentas, volvió a tener vigencia la denominada cirugía ambulatoria cuyo fundamento es permitir al paciente el retorno al hogar pocas horas después de realizado un procedimiento quirúrgico realizado con anestesia local o regional y hasta general, pero con cuidados postoperatorios sin internación (Cruz, 2020), acentuando así el menoscabo de este relación y de paso, la garantía de los derechos del paciente (Nasiłowski, 2007). Estas falencias se erigen como uno de los argumentos más relevantes, para que la consulta preanestésica sea un imperativo y no simplemente un trámite (Castañeda, 2019), pues la misma debe ser implementada no sólo como se realiza frecuentemente bajo la modalidad de simple visita preanestésica.

\section{Estándares de aplicación}

Como se ha mencionado, se parte del supuesto de la legitimidad y la legalidad del consentimiento como una forma de respeto a los derechos del paciente, de los cuales surgen deberes tales como la información debidamente ilustrada y la aceptación expresa de éste. En cuanto al contenido del consentimiento para que éste sea legítimo, se tiene que debe contener:

(i) Una descripción global de las técnicas de anestesia y sus modalidades, que pueden ser utilizadas (ASA, 2021) (Mutizábal, 2011), (Zaera, 2021); 
(ii) Una puntualización sobre la posibilidad de modificar la técnica prevista, para que no invalide el consentimiento por supuesto incumplimiento y ante la posibilidad de consecuencias legales en caso de incidentes o accidentes (Mutizábal, 2011) (Zaera, 2021), por ejemplo: seleccionar un bloqueo regional lumbar, pero ante la dificultad de realizarlo decidirse por anestesia general y viceversa, o cuando enfrentamos una vía aérea difícil que obligue a optar por un bloqueo locorregional;

(iii) Una explicación detallada que permita conocer al paciente aspectos como su vulnerabilidad dependiente de su estado de salud, el procedimiento propuesto, las alternativas terapéuticas, las probabilidades de éxito o fracaso del procedimiento, los efectos directos y colaterales a los que se expone, los beneficios probables del procedimiento, los cuidados en el pre, durante y pos procedimiento, ilustrada suficientemente sobre el principio fundamental de la bioética, en un espacio propicio para que el paciente pueda formular las preguntas que considere y obtener las respuestas adecuadas por parte del anestesiólogo.

Se debe tener presente que la redacción de un consentimiento legítimo para anestesiología debe ser diferente que el confeccionado para el uso de fármacos o para procedimientos quirúrgicos, por tanto, será único ya que anestesiología tiene sus propios riesgos y consecuencias que lo diferencias de otras prácticas en salud, para lo cual se recomienda, así está establecido en una parte importante de las legislaciones-, que se realice por escrito (Christen et al. 2014).

Por lo anterior, y como una forma de aportar a la solución de la problemática evidenciada, se propone un documento que sirva de modelo guía para las prácticas anestésicas, el cual tiene como características, que reúne los postulados para configurar un consentimiento en términos de legitimidad y legalidad, a partir de aspectos bioéticos y jurídicos.

Esta propuesta que se adjunta a este artículo, recomienda informar e ilustrar sobre el estado del paciente, el procedimiento propuesto, las alternativas terapéuticas, las probabilidades de éxito o fracaso del procedimiento, los efectos directos y colaterales a 
los que se expone, los beneficios probables del procedimiento y los cuidados que se otorgará en el pre, durante y pos procedimiento.

\section{CONCLUSIONES}

La anestesia es un acto médico, complejo y especializado, que no está exento de riesgos. Como toda intervención farmacológica e instrumental, es capaz de producir secuelas en el organismo que van desde efectos leves hasta consecuencias fatales. En tal sentido, es indispensable que el paciente reciba toda la información sobre estas particularidades antes de firmar su consentimiento y sujetarse a la normativa sanitaria establecida por los códigos internacionales de ética médica.

En la práctica clínica de la especialidad, se advierte que la firma del consentimiento no se cumple a cabalidad debido a circunstancias como: diferencia entre legislaciones de los países en donde se aplica, variación entre formularios utilizados en cuanto al tipo de información y lenguaje, y una débil relación médico-paciente entre el anestesiólogo y el candidato a intervención.

Si el acto médico es un procedimiento de carácter legal también lo es la firma del documento de aceptación que debería denominarse consentimiento legítimo en vez de consentimiento informado, cuyo contenido, como una manera de respetar los derechos del paciente y conceder mayor solvencia al tratamiento anestésico-quirúrgico.

Este documento contentivo de un consentimiento legítimo, debe incorporar como mínimo (i) La descripción global de lo que significa el acto anestésico en términos de una información debidamente ilustrada (ii) La puntualización sobre la modificación que puede requerir un procedimiento anestésico durante su curso (iii) La explicación detallada los pormenores del procedimiento en el pre, trans y postoperatorio según el estado de vulnerabilidad del paciente (iv) Establecer los espacios adecuados para solventar todas las dudas que tenga el paciente sobre el acto anestésico antes de legitimar su consentimiento (v) Ser enfático en todo momento sobre el derecho del paciente a ejercer su autonomía (vi) El uso de un lenguaje compresible, evitando los tecnicismos médicos 
(vii) La aceptación debe surgir después de una amplia socialización de su contenido y (viii) Deberá ser firmado antes de llevar a cabo el acto anestésico como tal.

Se convierte en prioritaria le necesidad de estandarizar un formato de consentimiento legítimo dentro del ejercicio de la anestesiología y su aplicación debe hacerlo el especialista a través de una eficiente relación anestesiólogo-paciente condición que se cumple únicamente durante la consulta preanestésica.

\section{FINANCIAMIENTO}

No monetario.

\section{AGRADECIMIENTO}

A la Universidad Católica de Cuenca; a la Maestría en Derecho Médico: por apoyar el desarrollo de esta investigación.

\section{REFERENCIAS CONSULTADAS}

ASA. (2016). Guidelines for Patient Care in Anesthesiology. Committee of Origin: Surgical Anesthesia. Washington, DC: American Society of Anestehsiologists.

ASA. (2021). American Society of Anesteshiologists. https://www.asahq.org/

Asamblea Nacional de Montecristi. (2008). Constitución Nacional del Ecuador. Montecristi: Ministerio de Gobierno.

Castañeda, M. (2019). Consulta preanestésica, ¿un trámite incómodo o una necesidad obligatoria? [Pre-anesthetic consultation, an uncomfortable procedure or a mandatory need?]. Recuperado de https://n9.cl/2ilm1

Chrimes, N., \& Marshall, S. D. (Jan de 2018). The illusion of informed consent. Anaesthesia, 73(1), 9-14.

Christen, M., Ineichen, C., \& Tanner, C. (2014). How "moral" are the principles of biomedical ethics?--a cross-domain evaluation of the common morality hypothesis. BMC Med Ethics, 15(47). 
CIDH. (2020). Cuadernillo de Jurisprudencia de la Corte Interamericana de Derechos Humanos No. 28: Derecho a la salud [Case Law of the Inter-American Court of Human Rights No. 28: Right to Health]. San José, C. R.: Corte Interamericana de Derechos Humanos y Cooperación Alemana (GIZ). https://www.corteidh.or.cr/sitios/libros/todos/docs/cuadernillo28.pdf

Cocanour, C. S. (2017). Informed consent-It's more than a signature on a piece of paper. Am J Surg, 214(6), 993-7.

Cordero, I. (2011). El riesgo de la anestesia [The risk of anesthesia]. Revista Cubana de Anestesiología y Reanimación, 10(2), 82-3.

Córdoba, R. (2010). Ciencias Políticas, Bioética y Bioderecho [Political Science, Bioethics and Biolaw]. Obtenido de https://n9.cl/xbps1

Cruz, J. (2020). Cirugía mayor ambulatoria: antecedentes, evolución y logros [Major outpatient surgery: antecedents, evolution and achievements]. Gac Méd Espirit, 22(1), 37-48.

De la Maza Gazmuri, I. (2010). Consentimiento informado, una visión panorámica [Informed consent, an overview]. lus et Praxis, 16(2), 89-120.

Dobler, L. (2001). Aspectos legales y éticos del consentimiento informado en la atención médica en México [Legal and ethical aspects of informed consent in medical care in Mexico]. Rev Mex Patol Clin, 48(1), 4-6.

Duarte, J. \& Parra, Eglée. (2014). Lo que debes saber sobre un trabajo de investigación [What you should know about a research paper]. 3ra edición. Maracay: Freddy Morlés

Fleisher, L. (2016). Riesgo de la anestesia [Risk of anesthesia]. En R. Miller, Anestesia (págs. 735-62). Barcelona: Elsevier.

FME. (2021). Ley de Federación Médica Ecuatoriana [Ecuadorian Medical Federation Law]. Recuperado de https://n9.cl/u9lgi

Forrellat-Barrios, M. (2014). Calidad en los servicios de salud: un reto ineludible [Quality in health services: an unavoidable challenge]. Revista Cubana de Hematología, Inmunología y Hemoterapia, 30(2), 179-83. 
García, R. S. (2001). La responsabilidad penal del médico. México: Porrúa.

Gonzáles, Q. E. (2004). Consentimiento informado, una realidad de nuestros tiempos [Informed consent, a reality of our times]. Aten Fam, 11(3), 1-3.

Haller, G., Laroche, T., \& Clergue, F. (2011). Morbidity in anaesthesia: today and tomorrow. Best practice \& research. Clinical anaesthesiology, 25(2), 123-132. https://doi.org/10.1016/j.bpa.2011.02.008

Hernández-Cortez, E. (2017). Seguridad en Anestesiología [Safety in Anesthesiology]. Anestesia en México, 29(2), 1-2., 29(2), 1-2.

Kounis, N. G., Koniari, I., Tzanis, G., Soufras, G. D., Velissaris, D., \& Hahalis, G. (2020). Anaphylaxis-induced atrial fibrillation and anesthesia: Pathophysiologic and therapeutic considerations. Annals of cardiac anaesthesia, 23(1), 1-6. https://doi.org/10.4103/aca.ACA 10019

Lagasse, R. (2002). Anesthesia safety: model or myth? A review of the published literature and analysis of current original data. Anesthesiology, 97, 1609-17.

Ley de Derechos y Amparo de los Pacientes. Registro Oficial Suplemento 626 de 03-feb.1995 Ultima modificación: 22-dic.-2006. https://n9.cl/x5xn

Lin, S. T., Ohbayashi, M., Yamamoto, T., Onimaru, H., \& Kogo, M. (2017). Effects of riluzole on spinal seizure-like activity in the brainstem-spinal cord preparation of newborn rat. Neurosci Res, 125, 46-53.

Luethi, D., \& Liechti, M. (2020). Designer drugs: mechanism of action and adverse effects. Arch Toxicol, 94(4), 1085-1133.

Mainetti, J. A. (1989). Ética médica. La Plata, Argentina: Quirón.

Mallardi, V. (2005). The origin of informed consent. Acta Otorhinolaryngol Ital, 25(5), 31227.

McCann, M. E., \& Soriano, S. G. (2019). Does general anesthesia affect neurodevelopment in infants and children?. BMJ (Clinical research ed.), 367, 16459. https://doi.org/10.1136/bmj.16459 
Mellin-Olsen, J., \& Staender, S. (2014). The Helsinki Declaration on Patient Safety in Anaesthesiology: the past, present and future. Current opinion in anaesthesiology, 27(6), 630-634. https://doi.org/10.1097/ACO.0000000000000131

Mena, RM, \& Rodríguez-Mota E. (2014). Valoración preanestésica. Importancia en el paciente quirúrgico [Preanesthetic evaluation. Importance in the surgical patient]. Rev Hosp Jua Mex, 81(3):193-198. https://n9.cl/4nprp

Mesa, P., Santotoribio, J. D., Ramos, V., González, M. Á., Pérez, S., \& Portilla, D. (2016). Daño cerebral postanestesia general [Post general anesthesia brain damage]. Med Clin, 146(9), 384-8.

Mikstacki, A., Zakerska-Banaszak, O., Skrzypczak-Zielinska, M., Tamowicz, B., Szalata, M., \& Slomski, R. (2015). Glutathione S-transferase as a toxicity indicator in general anesthesia: genetics and biochemical function. J Clin Anesth, 27(1), 73-9.

Miranda-Novales, M, \& Villasís-Keever, M. (2019). El protocolo de investigación VIII. La ética de la investigación en seres humanos [Research protocol VIII. The ethics of research on human subjects]. Revista alergia México, 66(1), 115122. https://doi.org/10.29262/ram.v66i1.594

Moctezuma, B. G. (2002). Derechos y deberes de los pacientes [Rights and duties of patients]. Rev Conamed, 7(2), 35-40.

Mutizábal, G. (2011). Consentimiento informado y anestesia [Informed consent and anesthesia]. Rev Chil Anest, 40, 77-102.

Narro-Robles, J, Rodríguez-Domínguez, J, Viesca-Treviño, C, \& Abreu-Hernández, L. (2004). Ética y salud: Retos y reflexiones [Ethics and health. Challenges and Reflections]. Gaceta médica de México, 140(6), 661-666.

Nasiłowski, W. (2007). Patient's consent to treatment with reference to the development of medical ethics. Wiad Lek, 60(3-4), 198-200.

Núñez de Villavicencio, P. (2006). Consentimiento educado vs Consentimiento informado [Educated consent vs Informed consent]. Rev Cubana Salud Pública, 32(4). 
Osler, W. (1913). Evolution of Modern Medicine: A Series of Lectures Delivered at Yale University on the Silliman Foundation in April, 1913 (6th ed., Vol. ISBN 1628450118). Kaplan Publishing.

Pardina, B., Metje, T., \& Villalonga, A. (2001). Embarazo y parto en la mujer con lesión medular en fase crónica [Pregnancy and childbirth in women with chronic spinal cord injury]. Rev Esp Anestesiol Reanim, 48(2), 93-6.

Pinto, B., \& Díaz, R. (2013). Asentimiento y consentimiento informado en pediatría: aspectos bioéticos y jurídicos en el contexto colombiano [Assent and informed consent in pediatrics: bioethical and legal aspects in the Colombian context]. Revista Colombiana de Bioética, 8(1), 144-65.

Ramírez, E., Coronado, G., \& Alfaro, M. (2006). Helsinki: consideraciones finales. Rev Filosofía Univ Costa Rica, XLIV(111-112), 185-92.

Rose, R. (1986). Informed consent: history, theory, and practice. Am J Otol, 7(1), 82-5. Schönenberger, S., Hendén, P. L., Simonsen, C. Z., Uhlmann, L., Klose, C., Pfaff, J., Yoo, A. J., Sørensen, L. H., Ringleb, P. A., Wick, W., Kieser, M., Möhlenbruch, M. A., Rasmussen, M., Rentzos, A., \& Bösel, J. (2019). Association of General Anesthesia vs Procedural Sedation With Functional Outcome Among Patients With Acute Ischemic Stroke Undergoing Thrombectomy: A Systematic Review and Meta-analysis. JAMA, 322(13), https://doi.org/10.1001/jama.2019.11455

Sivkov, A., Golovanov, S., \& Zhukova, L. (2019). Prediction of the effectiveness of the therapy of LUTS/BPH by Serenoa repens extracts. Urologiia, 3, 14-22.

Spector, L. R., Madigan, L., Rhyne, A., Darden, B., \& Kim, D. (2008). Cauda equina syndrome. J Am Acad Orthop Surg, 16(8), 471-9.

Stain, S. C. (2016). Informed Surgical Consent. J Am Coll Surg, 222(4), 717-8.

Taylor, R. M. (2013). Ethical principles and concepts in medicine. Handb Clin Neurol, 118, $1-9$.

Torres, M. (2011). La transformación de la ética médica [The transformation of medical ethics]. Sanidad Militar, 67(2), 131-134. 
Iustitia Socialis. Revista Arbitrada de Ciencias Jurídicas

Año VI. Vol. VI. N 1. Edición Especial. 2021

Hecho el depósito de Ley: FA2016000064

ISSN: 2542-3371

FUNDACIÓN KOINONIA (F.K). Santa Ana de Coro, Venezuela

Marcos Antonio Portilla-Kirby; Andrés Esteban Jaimes-Grimaldos

Waisel, D. B., Lamiani, G., Sandrock, N. J., Pascucci, R., Truog, R. D., \& Meyer, E. C. (2009). Anesthesiology trainees face ethical, practical, and relational challenges in obtaining informed consent. Anesthesiology, 110(3), 480-86.

Wheeler, R. (2017). The evolution of informed consent. Br J Surg, 104(9), 1119-20.

Wilkinski, J. A., \& Piaggio, A. N. (1993). El consentimiento informado. Primera parte: el deber de informar [Informed consent. Part one: the duty to inform]. Rev Arg Anest, 51(2), 87-105.

Zaera, L. (2021). Consentimiento informado en Anestesia, un deber y un derecho [Informed consent in anesthesia, a duty and a right]. Recuperado de https://n9.cl/lc4rn

Zarnegar, R. (2018). Informed consent in anaesthesia. Br J Anaesth, 120(4), 883-4.

Zheng, H. X., Eric Nyam, T. T., Liu, C. A., Lee, Y. L., Kuo, J. R., \& Sung, K. C. (2020). Spontaneous Spinal Epidural Hematoma After Normal Spontaneous Delivery with Epidural Analgesia: Case Report and Literature Review. World neurosurgery, 137, 214-217. https://doi.org/10.1016/j.wneu.2020.01.240

Zúñiga, C., \& Zúñiga-Hernández, J. (Aug de 2019). Exception to informed consent in research: when is this possible in Chile? Rev Med Chil, 147(8), 1039-45.

C2021 por los autores. Este artículo es de acceso abierto y distribuido según los términos y condiciones de la licencia Creative Commons Atribución-NoComercial-Compartirlgual 4.0 Internacional (CC BY-NC-SA 4.0)

(https://creativecommons.org/licenses/by-nc-sa/4.0/). 